\section{(C) OPEN ACCESS}

\title{
Flortaucipir tau PET imaging in semantic variant primary progressive aphasia
}

\author{
Sara J Makaretz, ${ }_{1}^{1}$ Megan Quimby, ${ }_{1}^{1}$ Jessica Collins, ${ }^{1}$ Nikos Makris, ${ }^{2}$ Scott McGinnis, ${ }^{1,3}$ \\ Aaron Schultz, ${ }^{4,5}$ Neil Vasdev, ${ }^{6}$ Keith A Johnson, ${ }^{3,5,6}$ Bradford C Dickerson ${ }^{1,4,5}$
}

${ }^{1}$ Frontotemporal Disorders Unit, Department of Neurology, Massachusetts General Hospital and Harvard Medical School, Charlestown, Massachusetts, USA

${ }^{2}$ Center for Morphometric Analysis, Department of Psychiatry, Massachusetts General Hospital and Harvard Medical School, Charlestown, Massachusetts, USA ${ }^{3}$ Center for Alzheimer Research and Treatment, Department of Neurology, Brigham and Women's Hospital, Boston, Massachusetts, USA

${ }^{4}$ Martinos Center for Biomedical Imaging, Massachusetts General Hospital and Harvard Medical School, Charlestown, Massachusetts, USA

${ }^{5}$ Alzheimer's Disease Research Center, Department of

Neurology, Massachusetts General Hospital and Harvard Medical School, Boston, Massachusetts, USA ${ }^{6}$ Division of Nuclear Medicine and Molecular Imaging, Department of Radiology, Massachusetts General Hospital and Harvard Medical School Boston, Massachusetts, USA

\section{Correspondence to}

Dr Bradford C Dickerson, MGH Frontotemporal Disorders Unit, 149 13th St. Suite 2691

Charlestown, MA 02129, USA brad.dickerson@mgh.harvard. edu

Received 10 May 2017 Revised 7 July 2017

Accepted 18 July 2017 Published Online First 6 October 2017

\section{SLinked}

- http://dx.doi.org/10.1136/ jnnp-2017-316402

Check for updates

To cite: Makaretz SJ Quimby M, Collins J, et al.J Neurol Neurosurg Psychiatry 2018:89:1024-1031.

\section{ABSTRACT}

Objective The semantic variant of primary

progressive aphasia (svPPA) is typically associated with frontotemporal lobar degeneration (FTLD) with longTAR DNA-binding protein (TDP)-43-positive neuropil threads and dystrophic neurites (type $\mathrm{C}$ ), and is only rarely due to a primary tauopathy or Alzheimer's disease. We undertook this study to investigate the localisation and magnitude of the presumed tau Positron Emission Tomography (PET) tracer $\left[{ }^{18} \mathrm{~F}\right] \mathrm{Flortaucipir}$ (FTP; also known as T807 or AV1451) in patients with SVPPA, hypothesising that most patients would not show tracer uptake different from controls.

Methods FTP and $\left[{ }^{11} \mathrm{C}\right]$ Pittsburgh compound B PET imaging as well as MRI were performed in seven patients with svPPA and in 20 controls. FTP signal was analysed by visual inspection and by quantitative comparison to controls, with and without partial volume correction.

Results All seven patients showed elevated FTP uptake in the anterior temporal lobe with a leftward asymmetry that was not observed in healthy controls. This elevated FTP signal, largely co-localised with atrophy, was evident on both visual inspection and quantitative cortical surface-based analysis. Five patients were amyloid negative, one was amyloid positive and one has an unknown amyloid status.

Conclusions In this series of patients with clinical profiles, structural MRI and amyloid PET imaging typical for sVPPA, FTP signal was unexpectedly elevated with a spatial pattern localised to areas of atrophy. This raises questions about the possible off-target binding of this tracer to non-tau molecules associated with neurodegeneration. Further investigation with autopsy analysis will help illuminate the binding target(s) of FTP in cases of suspected FTLD-TDP neuropathology.

\section{INTRODUCTION}

The semantic variant of primary progressive aphasia (svPPA) is a form of PPA with well-characterised, relatively stereotypical clinical features initially manifesting as a fluent, anomic aphasia with prominent semantic memory loss associated with asymmetrical (usually dominant hemisphere) anterior temporal cortical atrophy. ${ }^{1-4}$ In more than $90 \%$ of cases with this characteristic clinic-anatomical phenotype, the underlying neuropathology is frontotemporal lobar degeneration (FTLD) with long TDP-43-positive neuropil threads and dystrophic neurites in temporal and frontal cortices (known as TDP-43 type C pathology). ${ }^{56}$ Rarely, the primary neuropathology of svPPA is a tauopathy-usually
Alzheimer's disease (AD) or Pick's disease, ${ }^{7-9}$ or as has recently been reported, globular glial tauopathy. $^{10}$

The advent of tau PET imaging has revolutionised our ability to measure neurofibrillary paired helical filament (PHF) tau pathology in AD, but findings with these ligands in non-Alzheimer tauopathies with straight filament tau are less clear. To date, small in vivo series have been reported using the presumptive tau PET ligand flortaucipir (FTP) in patients with clinical diagnoses of progressive supranuclear palsy, Microtubule-associated protein tau (MAPT)-related FTLD and corticobasal syndrome, ${ }^{11-13}$ suggesting that tracer uptake is present in areas of expected neurodegeneration. However, autoradiographic and biochemical studies of postmortem tissue from patients with non-AD tauopathies have shown weak or no evidence of FTP binding to straight filament tau, and no binding to TDP inclusions. ${ }^{14-17}$

We undertook this study to investigate the uptake, localisation and magnitude of the putative tau PET tracer FTP in a series of patients with svPPA. Since the majority of patients with svPPA have TDP-43 pathology, we hypothesised that the majority of our sample will have non-tau pathology and exhibit FTP signal no different from controls, and have little or no evidence of cortical amyloid plaques. Although far less likely, our sample may include patients with svPPA due to AD or a primary tauopathy. If a patient in our sample has svPPA due to AD pathology, we expected to see evidence of cortical amyloid, and FTP binding localised congruently with atrophy and at a magnitude consistent with what has been observed in other reports of patients with atypical AD. ${ }^{18} 19$ If a patient has svPPA due to a primary tauopathy such as Pick's disease, we expected little or no evidence of cortical amyloid, and low FTP binding consistent with postmortem findings of low or no FTP affinity for straight filament tau. Alternatively, if FTP binds to a non-specific marker of neurodegeneration, we would expect increased FTP signal in all of the patients with svPPA, co-localised with atrophy.

\section{METHODS}

\section{Participants}

svPPA Patients

Seven patients with svPPA were recruited from an ongoing longitudinal study being conducted in the Primary Progressive Aphasia Program of the Massachusetts General Hospital (MGH) Frontotemporal 
Table 1 Demographic and clinical characteristics of participants

\begin{tabular}{|c|c|c|c|c|c|c|c|c|c|c|c|}
\hline & \multirow[b]{2}{*}{ Age } & \multirow[b]{2}{*}{ Sex } & \multirow{2}{*}{$\begin{array}{l}\text { Illness } \\
\text { duration }\end{array}$} & \multirow{2}{*}{$\begin{array}{l}\text { PiB } \\
\text { (FLR DVR) }\end{array}$} & \multirow[b]{2}{*}{ MMSE* } & \multicolumn{3}{|l|}{ CDR } & \multicolumn{2}{|l|}{ PASS } & \multirow[b]{2}{*}{ BNT } \\
\hline & & & & & & Global & Behaviour & Language & SOB & Single word & \\
\hline Case 1 & 72 & $\mathrm{~F}$ & 10 years & 1.05 & $23 / 30$ & 0.5 & 0 & 1 & 3 & 1 & 1 \\
\hline Case 2 & 66 & $\mathrm{M}$ & 8 years & $t$ & $14 / 30$ & 1 & 1 & 2 & 13.5 & 2 & 1 \\
\hline Case 3 & 71 & $\mathrm{~F}$ & 3 years & $\ddagger$ & $23 / 30$ & 0.5 & 0 & 1 & 3.5 & 1 & 1 \\
\hline Case 4 & 54 & $\mathrm{~F}$ & 6 years & 1.04 & $24 / 30$ & 0.5 & 0.5 & 0.5 & 4.5 & 0.5 & 2 \\
\hline Case 5 & 53 & M & 4 years & 1.06 & $20 / 30$ & 0.5 & 1 & 1 & 7.5 & 1 & 1 \\
\hline Case 6 & 63 & $\mathrm{~F}$ & 6 years & 1.07 & $25 / 30$ & 0.5 & 0 & 1 & 5 & 1 & 3 \\
\hline Case 7 & 80 & $M$ & 6 years & 1.49 & $19 / 30$ & 0.5 & 0.5 & 1 & 7 & 0.5 & 4 \\
\hline $\begin{array}{l}\text { svPPA } \\
n=7\end{array}$ & $66.0 \pm 9.6$ & $4 \mathrm{~F}$ & $6.6 \pm 2.5$ & $1.14 \pm 0.19$ & $21.1 \pm 3.8$ & & & & & & \\
\hline $\begin{array}{l}\text { Control } \\
n=20\end{array}$ & $62.8 \pm 8.8$ & $9 \mathrm{~F}$ & - & $1.09 \pm 0.05$ & $29.5 \pm 0.6$ & & & & & & \\
\hline
\end{tabular}

Group values for age, PiB, FLR DVR and MMSE are mean \pm SD.

*Significant difference between svPPA and control groups, $\mathrm{p}<0.001$.

† Case 2 did not complete PiB PET nor was CSF available.

¥ Case 3 had CSF amyloid beta and phospho-tau levels consistent with the absence of cerebral amyloid plaques and neurofibrillary tangles.

BNT, Boston Naming Test summary score (\# correct, spontaneous or with semantic cue); CDR, Clinical Dementia Rating; FLR DVR, distribution volume ratio of FLR regions (ie, frontal, lateral parietal and temporal, and retrosplenial cortices); MMSE, Mini-Mental State Examination; PASS, Progressive Aphasia Severity Scale; PiB PET, Pittsburgh compound B PET; single word, PASS single word comprehension score; SOB, PASS Sum of Boxes score; svPPA, semantic variant of primary progressive aphasia.

Disorders Unit (see table 1). The patients were all right-handed, native English speakers. All patients with svPPA were evaluated using a structured clinical assessment performed by a behavioural neurologist and speech pathologist, as previously described, including rating using the Progressive Aphasia Severity Scale. ${ }^{120}$ All participants gave written informed consent in accordance with the institutional review board of the MGH and Partners Healthcare System Human Research Committee.

Patients were diagnosed with svPPA based on consensus guidelines. ${ }^{2}$ A diagnosis of PPA required progressive deterioration of speech and/or language functions, and that deficits be largely restricted to speech and/or language for at least the first 2 years of the illness. ${ }^{21}$ Each of the patients with svPPA included in this study had prototypical clinical (semantic language impairments) and anatomical (cortical atrophy that was most prominent in the left anterior temporal lobes) characteristics on first assessment, and was at a mild stage of overall clinical impairment at time of scanning. Visual inspection of a clinical MRI ruled out other causes of focal brain damage and in all cases provided imaging support for the diagnosis (anterior temporal atrophy).

\section{Controls}

Imaging data from 20 age-matched and gender-matched, righthanded, cognitively and neurologically normal controls were included. All 20 individuals underwent detailed clinical and cognitive assessments and were determined to be cognitively normal. Amyloid PET scanning demonstrated minimal or no evidence of elevated amyloid, with low or minimal amyloid defined as $\left[{ }^{11} \mathrm{C}\right]$ Pittsburgh compound B (PiB) distribution volume ratio (DVR) of FLR regions (ie, frontal, lateral parietal and temporal, and retrosplenial cortices) $<1.2 .^{22}$ Age, gender, PiB FLR and MiniMental State Examination (MMSE) of controls are summarised in table 1 .

\section{Neuroimaging data acquisition and analysis Magnetic resonance imaging}

All participants (patients and controls) underwent a 3T MRI scan (Siemens TIM Trio 3.0T, Siemens Medical Systems, Erlingan, Germany) that included acquisition of T1-weighted multi-echo magnetisation prepared rapid acquisition gradient echo (MPRAGE) structural images. The MRI analysis methods employed here have been previously described in detail, including cortical thickness processing and spherical registration to align subjects' cortical surfaces (FreeSurfer stable release V. 6.0.0, http://surfer.nmr.mgh.harvard.edu). ${ }^{23}$

\section{PET}

All seven patients also underwent FTP, and five of the seven underwent $\left[{ }^{11} \mathrm{C}\right] \mathrm{PiB}$ imaging. Both FTP and $\left[{ }^{11} \mathrm{C}\right] \mathrm{PiB}$ imaging were acquired for all 20 control subjects. FTP was prepared at $\mathrm{MGH}$ with a radiochemical yield of $14 \% \pm 3 \%$ and specific activity of $216 \pm 60 \mathrm{GBq} / \mu \mathrm{mol}(5837 \pm 1621 \mathrm{mCi} / \mu \mathrm{mol})$ at the end of synthesis $(60 \mathrm{~min})$, and validated for human use. ${ }^{24}\left[{ }^{11} \mathrm{C}\right]$ $\mathrm{PiB}$ was prepared as described previously ${ }^{17}$. All PET data were acquired using a Siemens/CTI (Knoxville, Tennessee) ECAT $\mathrm{HR}+$ scanner (3D mode; 63 image planes; $15.2 \mathrm{~cm}$ axial field of view; $5.6 \mathrm{~mm}$ transaxial resolution and $2.4 \mathrm{~mm}$ slice interval). FTP was acquired from 80 to $100 \mathrm{~min}$ after a $10.0 \pm 1.0 \mathrm{mCi}$ bolus injection in $4 \times 5$ min frames. Recent kinetic studies have shown that some regions may not reach steady state by $100 \mathrm{~min}$ postinjection $^{25}$; the standard acquisition window has since been increased for prospective subjects to $80-110 \mathrm{~min}$. $\left[{ }^{11} \mathrm{C}\right] \mathrm{PiB}$ PET was acquired with an 8.5 to $15 \mathrm{mCi}$ bolus injection followed immediately by a $60 \mathrm{~min}$ dynamic acquisition in 69 frames $(12 \times 15 \mathrm{~s}, 57 \times 60 \mathrm{~s})$. PET data were reconstructed and attenuation corrected, and each frame was evaluated to verify adequate count statistics; interframe head motion was corrected prior to further processing.

To evaluate the anatomy of cortical FTP and $\left[{ }^{11} \mathrm{C}\right] \mathrm{PiB}$ binding, each individual patient's PET data set was rigidly co-registered to the subject's MPRAGE MRI using SPM8 (Wellcome Department of Cognitive Neurology, Function Imaging Laboratory, London). Visual inspection confirmed accurate registration between anatomical and PET volumes. The cortical regions of interest (ROIs) defined by the FreeSurfer (FS) parcellation were transformed into the PET native space and PET data were sampled within each ROI. Similar to previous reports, ${ }^{19}$ FTP specific binding was expressed in FS ROIs as the standardised uptake value ratio (SUVR) using the cerebellar grey matter ROI as a reference. $\left[{ }^{11} \mathrm{C}\right] \mathrm{PiB}$ PET data were expressed in FS ROIs as the 
distribution volume ratio (DVR) with the cerebellar grey ROI as a reference, ${ }^{19}$ where regional time-activity curves (TAC) were used to compute regional DVRs for each ROI using the Logan graphical method applied to data from 40 to 60 min after injection. For surface-based analyses and ROI quantification, PET signal for each subject was sampled along the cortical ribbon and projected to the nearest surface vertex. Primary analyses of PET data were performed without partial volume correction using geometric transform matrix as implemented in FreeSurfer V. 6.0.0; analyses were repeated with partial volume correction as previously described. ${ }^{26}$

\section{Neuroimaging data analysis}

PET tracer uptake and cortical atrophy were assessed at both the individual patient and group levels. First, each patient's images were visually inspected. Then we created individual patient cortical surface maps of elevated FTP uptake and cortical thickness compared with the control group to visualise the spatial distribution of increased FTP in comparison to atrophy. For the individual atrophy map, we performed a surface-based, wholecortex general linear model (GLM) analysis using FreeSurfer, ${ }^{19}$ which compares the thickness of the individual patient's cortex at each vertex point across the entire cortical mantle with the group of controls. Similarly, we performed an GLM comparing FTP signal in the individual patient at each vertex point with FTP signal in the group of controls. In addition to the individual patient-level maps, we performed group-level GLMs comparing the entire svPPA group with controls, both for cortical atrophy and increased FTP signal relative to controls.

Finally, to further quantitatively compare the magnitude of FTP tracer uptake between patients and controls, we measured FTP signal within 34 FS ROIs per hemisphere from the Desikan-Killiany et al parcellation. ${ }^{27}$ We ran independent sample t-tests on five of these ROIs per hemisphere, selected to encompass areas with elevated FTP signal in the svPPA patient group as described below. In addition, we chose the precuneus ROI as a comparison region. To quantify the relationship between FTP signal and cortical thickness within each patient, we extracted mean FTP SUVR with and without partial volume correction, and cortical thickness for each of the 68 ROIs from the Desikan-Killiany parcellation (34 per hemisphere). A standardised t-score was calculated for cortical thickness within each ROI to represent the magnitude of cortical thickness within the ROI, normalised to the healthy control sample ((Patient cortical thickness $-\mu_{\text {con- }}$ trols $\left.) /\left(\sigma_{\text {controls }} \times \sqrt{ }((n+1) / n)\right)\right) .{ }^{28}$ Within each patient, Pearson correlation analyses (IBM SPSS Statistics V. 22) were performed for all ROIs to assess the relationship between FTP signal and cortical thickness t-scores ('atrophy'), using a method we previously published. ${ }^{19}$

\section{RESULTS}

Five of seven patients were amyloid negative based on amyloid PET, CSF or both. One patient's amyloid status was unknown due to inability to tolerate acquisition of a PiB scan or a CSF sample. One older patient with svPPA exhibited elevated PiB PET tracer uptake with a spatial distribution and magnitude (PiB FLR $=1.49$ ) consistent with the presence of cortical amyloid plaques. There were no significant differences between the control and svPPA groups with respect to age, gender or PiB FLR; the svPPA group had significantly lower MMSE scores. Table 1 provides details.

Surprisingly, visual inspection of FTP scans of each individual patient with svPPA showed asymmetrically elevated signal with a spatial distribution consistent with atrophy (see figure 1). The highest cortical signal was in the anterior temporal lobe (left $>$ right) with a variable degree of extension caudally in the ventral temporal cortex. Peak cortical FTP SUVR was $\geq 2.0$ in all but one subject with svPPA. Subject 6 showed a peak FTP SUVR left anterior temporal signal of 1.5 .

Slightly lower asymmetrical (left $>$ right) signal was seen in in the inferior temporal gyrus extending caudally in most patients to the caudal hippocampus or beyond, and in the frontoinsula and orbitofrontal cortex. In most patients, the SUVR of this signal was equal to or greater than 1.5 . In most patients, elevated

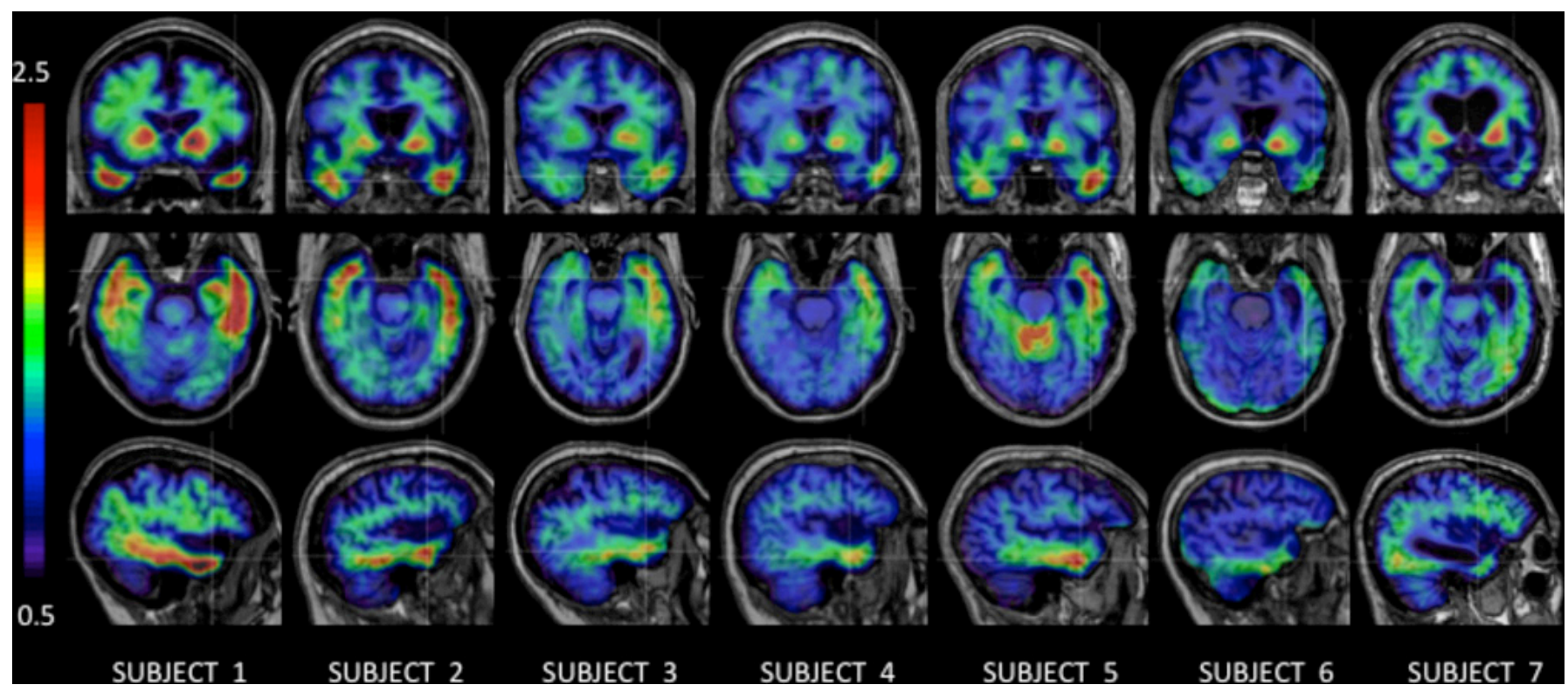

Figure 1 Flortaucipir standardised uptake value ratio overlaid on representative coronal, axial and sagittal MRI slices at levels that illustrate the primary regions of elevated signal in each case, which follows a stereotyped pattern largely co-localised with regional atrophy. These images are not partial volume corrected, and are displayed using a threshold range of 0.5-2.5. Cases 6 and 7 exhibit the lowest signal; case 7 is Pittsburgh compound B PET amyloid positive. 
signal was also seen in regions within the lateral temporal and parietal cortex, as well as ventrolateral frontal cortex, usually at FTP SUVR equal to or greater than 1.3. The posterior cingulate cortex showed peak FTP SUVR $<1.25$ in all but three patients (case $7: 1.7$; case $1: 1.5$; case $2: 1.3$ ).

In the patient with evidence of cortical amyloid plaques (figure 1, subject 7), FTP tracer uptake was localised similarly to the other cases, except that anterior temporal signal was more prominent in the anterior medial temporal region and that a few frontoparietal regions showed slightly elevated signal not seen in the other cases. Consistent with the other six cases, the magnitude of signal in this case was no greater than an SUVR of 2.0 in any region except the rostral ventral striatum in the region of the nucleus accumbens, where it was $\sim 2.2$ in the left hemisphere and $\sim 1.9$ in the right hemisphere. Most anterior temporal regions with elevated signal showed values in the range of 1.2-1.6. Elevated signal extended caudally in the ventral temporal lobe into posterior fusiform gyrus, greater in the left hemisphere $(\sim 1.7-1.8)$ than right $(\sim 1.4)$. Additionally, elevated signal was seen in left superior frontal gyrus and posterior cingulate cortex $(S U V R=1.7)$. This case also showed the highest signal of all the svPPA cases in the precuneus, slightly above that of controls, as described below.

Both patients and controls showed elevated signal within the basal ganglia, consistent with previous reports. ${ }^{29}{ }^{30}$ In patients only, elevated signal consistently extended at similar levels into the rostral ventral striatum, anterior to and distinct from the basal ganglia signal present both patients and controls. This rostral ventral striatal signal, in the vicinity of the nucleus accumbens, was highly lateralised (left $>$ right), consistent with lateralisation of cortical atrophy. This striatal signal was the most prominent brain signal in each patient, and peak FTP SUVR was $\geq 2.0$ in voxels within these regions. All subjects showed additional subcortical FTP binding near the substantia nigra of the midbrain, consistent with prior in vivo reports ${ }^{29}$ and postmortem reports of off-target FTP binding to neuromelanin-containing cells. ${ }^{1416}$

In all patients, elevated FTP signal was present in the cerebral cortical grey matter of the regions described and usually in the subjacent white matter, in some cases with most prominent signal at the grey-white junction. SUV images were inspected, and were confirmed to have the expected uniform, low uptake within the reference ROI. This increases our confidence that elevated FTP SUVR values in the svPPA cases is not due to choice of reference region. The exception to this is one subject with svPPA who exhibited focally increased FTP uptake in the anterior cerebellar grey matter $(\mathrm{SUVR}=2.1$; see figure 1 , subject 5$)$. This subject still exhibited high FTP SUVR values $(\sim 2.3)$ in the left anterior temporal lobe, with distribution and magnitude of FTP SUVR values similar to controls in occipital and parietal cortices.

Statistical comparison of each individual subject with controls demonstrated that most regions with signal $>1.3$ described above exhibited signal that was higher than the control group (see figure 2). Furthermore, the localisation of this elevated signal corresponded closely to regional atrophy. Each individual subject's atrophy map was strikingly similar to the individual subject map of FTP signal. A group map of elevated cortical FTP signal in svPPA compared with controls demonstrated similar findings (see figure 3 ). The spatial distribution of this elevated

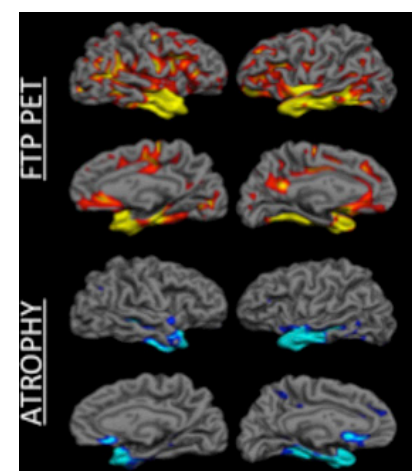

SUBJECT 1

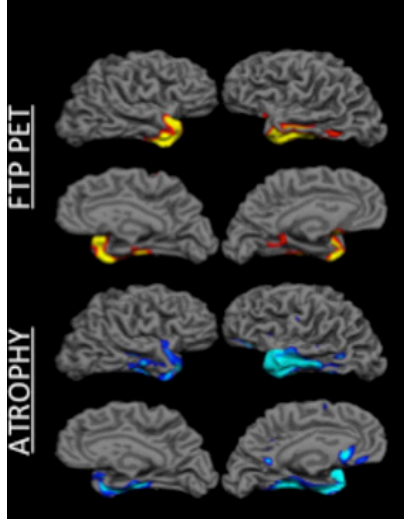

SUBJECT 5

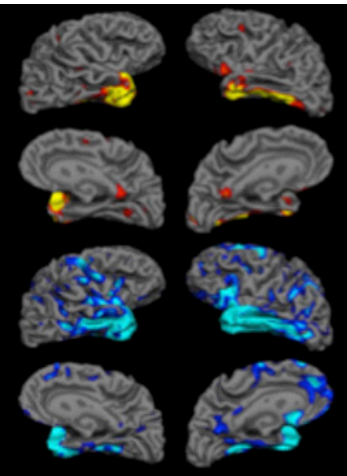

SUBJECT 2

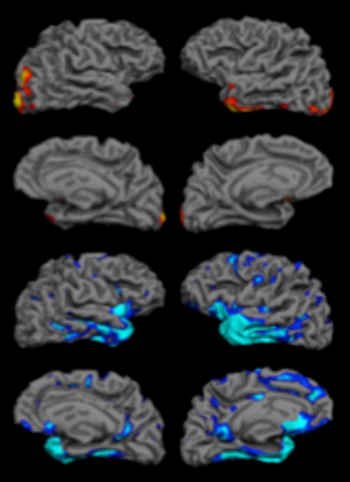

SUBJECT 6

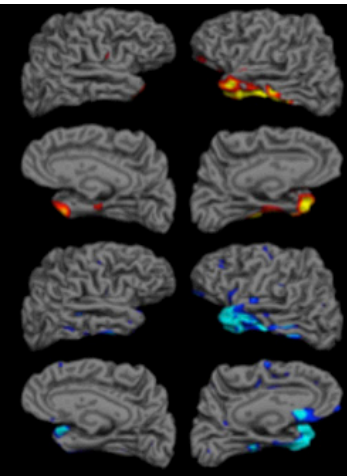

SUBJECT 3

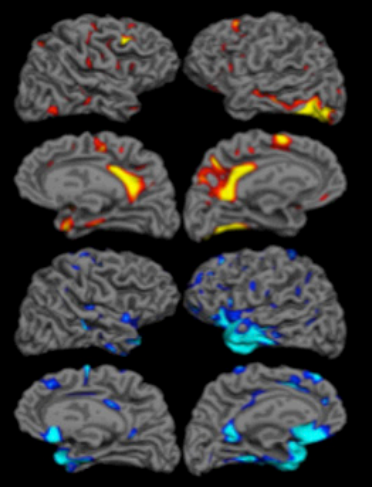

SUBJECT 7

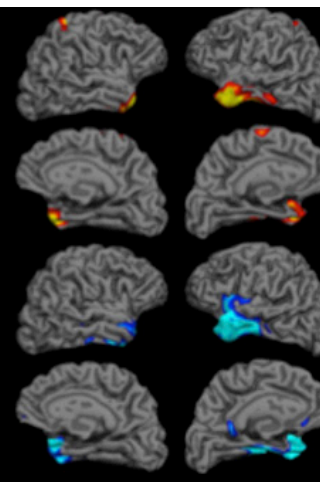

SUBJECT 4
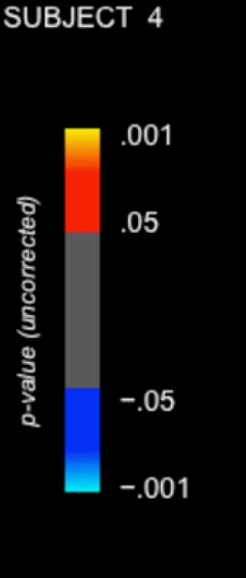

Figure 2 At the individual subject level, the distribution of increased FTP SUVR (red-yellow) and cortical atrophy (blue) are highly overlapping. Surface maps represent vertices with significant differences in measure of interest, as identified with t-tests comparing each subject to the control group. $p$ Values are uncorrected. For each subject, the top two rows show FTP SUVR images, while the bottom two rows show cortical atrophy. FTP, flortaucipir; SUVR, standardised uptake value ratio. 


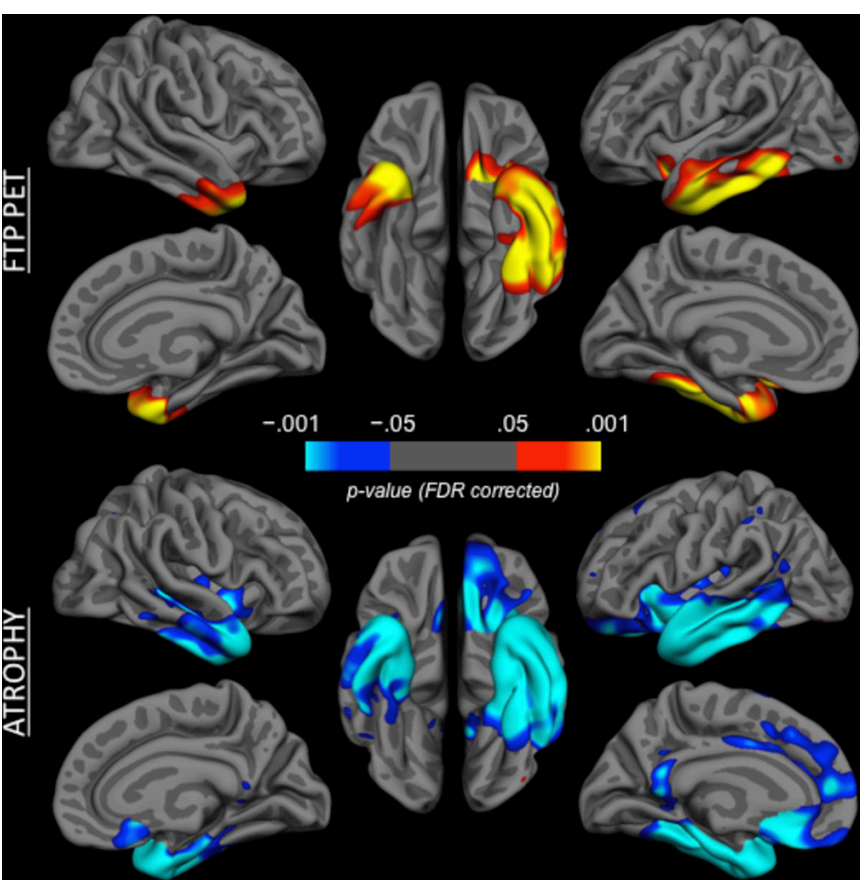

Figure 3 Group comparison of flortaucipir (FTP) standardised uptake value ratio versus controls (top, red-yellow) and cortical atrophy (bottom, blue). Coloured vertices on the cortical surface map indicate areas that are different in the semantic variant of primary progressive aphasia patient group versus the control group. Group comparisons are false discovery rate (FDR) corrected.

signal was highly consistent with the functional connectivity of the temporal pole, as we have previously demonstrated. ${ }^{1}$ Again, at the group level, the atrophy map (see figure 3) strongly overlapped with the map of elevated FTP signal. Few regions demonstrated atrophy without elevated FTP signal.

Within the Desikan-Killiany ROIs we selected for additional analysis, FTP signal was elevated in nearly all patients above the control group in the temporal pole, inferior temporal, middle temporal and fusiform cortex. FTP signal in the entorhinal cortex was elevated in 50\% of patients with svPPA. FTP signal in the precuneus was no different in patients with svPPA than in controls. Partial volume correction increases FTP signal substantially in all ROIs except precuneus. See figure 4 for an illustration of the localisation of the ROIs and for FTP signal.

Pearson correlation analyses across the 68 cortical Desikan-Killiany ROIs showed robust relationships between FTP SUVR and magnitude of cortical atrophy within each patient with svPPA (see figure 5). For each patient, correlation between cortical thickness t-score and FTP SUVR showed medium-to-large effects for both uncorrected FTP SUVR (case 1, -0.740 ; case 2, -0.572 ; case $3,-0.600$; case $4,-0.686$; case $5,-0.769$; case $6,-0.598$; case $7,-0.435)$ and partial volume corrected (PVC) FTP SUVR values (case $1,-0.779$; case $2,-0.627$; case 3 , -0.643 ; case $4,-0.733$; case $5,-0.771$; case $6,-0.568$; case 7, -0.661), where ROIs with higher FTP signal also showed more atrophy.

\section{DISCUSSION}

We were surprised to observe that, despite autoradiographic evidence of an absence of binding of FTP in postmortem tissue with FTLD TDP-43 pathology, each individual patient with svPPA in this series demonstrated elevated signal localised asymmetrically in the anterior temporal cortex and other areas of neurodegeneration. The absence of elevated amyloid biomarkers in all but one of these patients indicates that these patients do not likely have AD pathology with PHF tau inclusions. Therefore, the modest elevations in FTP co-localised and correlated with atrophy demonstrate either FTP binding to straight filament tau in the context of a primary tauopathy or non-specific binding co-localised with neurodegeneration in the context of TDP pathology. Based on the field's collective experience in clinicopathologic studies of patients with this syndrome (>90\% with TDP-43 pathology), it seems most likely that our findings point towards FTP binding to a molecule(s) associated with neurodegeneration other than tau. Some investigators have reported that certain forms of TDP-43 pathology bind to thioflavin stains in a manner suggestive of amyloid-like conformational properties, ${ }^{3132}$ thus offering the possibility that the in vivo signal we observe here is indicative of TDP-43, despite negative FTP autoradiography results with TDP-43 tissue. ${ }^{14} 16$

The spatial distribution of elevated FTP signal in each of the patients with svPPA in the present sample followed a highly stereotyped pattern, consistent with the localisation of typical neuropathology in svPPA or semantic dementia. In each case, the most prominent signal was present in the left temporal pole, inferior temporal gyrus and to a lesser degree middle temporal and fusiform gyri, regions known to be sites of prominent pathology and also known to be closely connected as part of a large-scale network. ${ }^{1}$ To varying degrees in each patient, FTP signal extended caudally into ventromedial and lateral anterior temporal cortical regions. Signal that was similarly localised but lower in magnitude was present in the right temporal pole and ventrolateral temporal cortex.

The localisation of FTP signal was highly similar to the localisation of regional atrophy in each individual patient as well as in group-wide analyses. This is quantitatively demonstrated by the robust correlations within each patient between increased regional FTP signal and more prominent regional atrophy. The localisation and magnitude of FTP are clearly different from the pattern seen in cognitively normal older adults with FTP binding-thought to represent either primary age-related tauopathy or early braakstage preclinical AD. ${ }^{29} 33$ Furthermore, it is distinct from that of prodromal $\mathrm{AD}$ or $\mathrm{AD}$ dementia, ${ }^{18} 1929$ and that of dementia with Lewy bodies. ${ }^{34}$ Yet in most patients with svPPA in this study, the magnitude of signal, even after partial volume correction, is substantially below that seen in patients with likely $\mathrm{AD}$ pathology. Interestingly, FTP signal within the precuneus was not elevated in all but one of these patients; it was slightly elevated in the patient with evidence of cerebral amyloid. This may suggest the presence of atypical AD pathology in a patient with svPPA, or dual FTLD-TDP and AD pathology as we have recently reported. ${ }^{3}$ We favour the latter, because the magnitude of FTP signal in this patient is substantially below the magnitude reported in cases of mild dementia driven primarily by $\mathrm{AD}$ pathology.

Although spatial resolution limits our ability to reliably distinguish FTP signal in the nucleus accumbens from signal in adjacent striatal structures, all seven patients demonstrated asymmetrically (left $>$ right) elevated signal in the rostral ventromedial striatum in the vicinity of the nucleus accumbens, an area of known pathology in svPPA. ${ }^{35}$ This signal appears to be distinct from high FTP signal seen in more caudal basal ganglia structures that has been reported in both healthy and patient samples. ${ }^{18} 29$

The major limitation of this study is the lack of autopsy follow-up to confirm the neuropathological diagnosis in these cases and the inability to perform tissue studies that would elucidate the possible binding target for FTP in cases with established non-PHF tau pathology. Abundant evidence indicates that the 


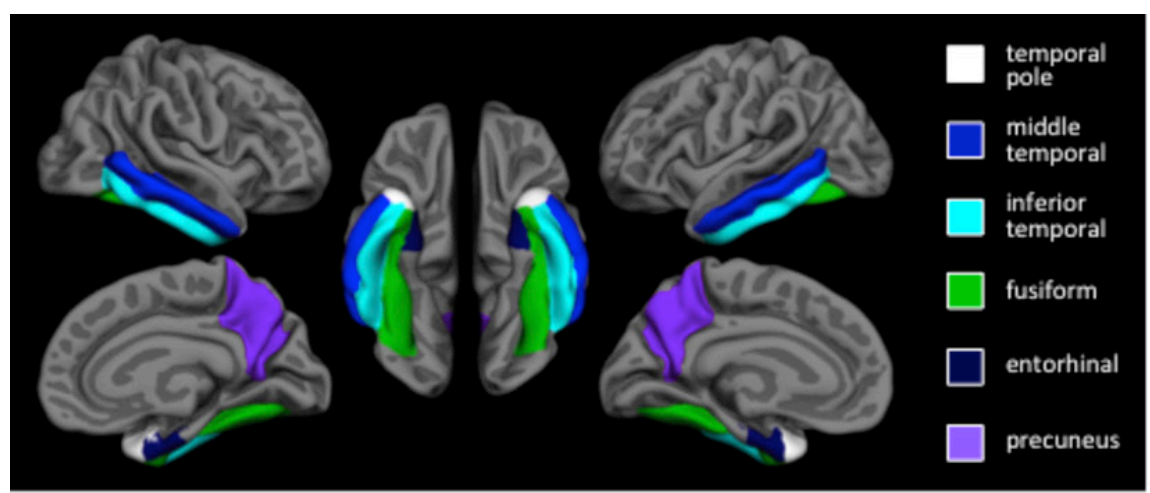

$\diamond$ SVPPA -

amyloid negative

$\diamond$ SVPPA .

amyloid positive

- SVPPA -

amyloid status

unknown

- control
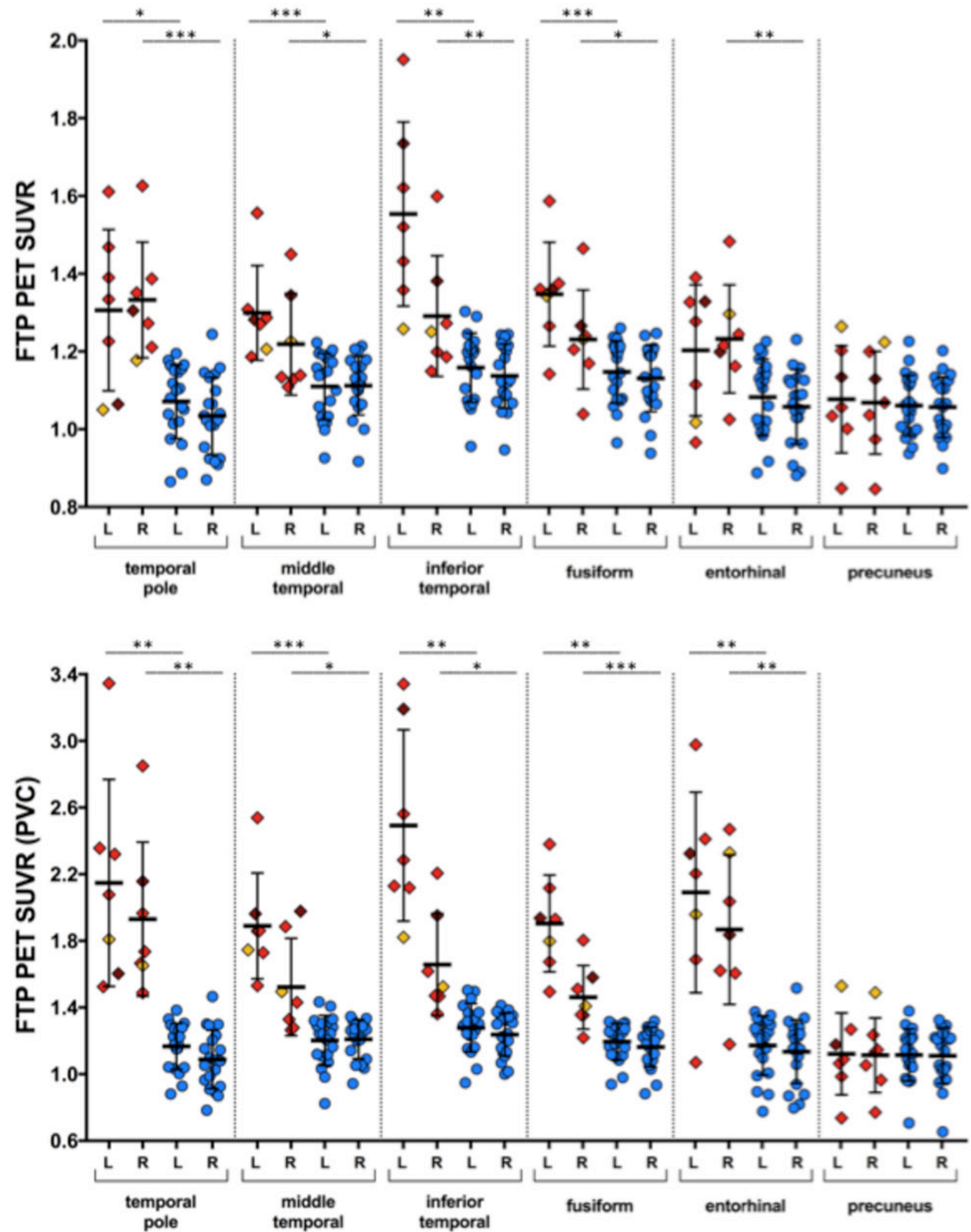

Figure 4 Region of interest measures of FTP SUVR magnitude, with and without partial volume correction. Cortical surface map shows regions of interest from the standard FreeSurfer parcellation. Statistical comparisons are indicated with top left bar comparing left hemisphere regions of interest between sVPPA and controls, and lower right bar comparing right hemisphere regions of interest; ${ }^{*} p<0.05$; ${ }^{* *} p<0.01$; ${ }^{* * *} p<0.001$. FTP, flortaucipir; SUVR, standardised uptake value ratio; svPPA, semantic variant of primary progressive aphasia. 


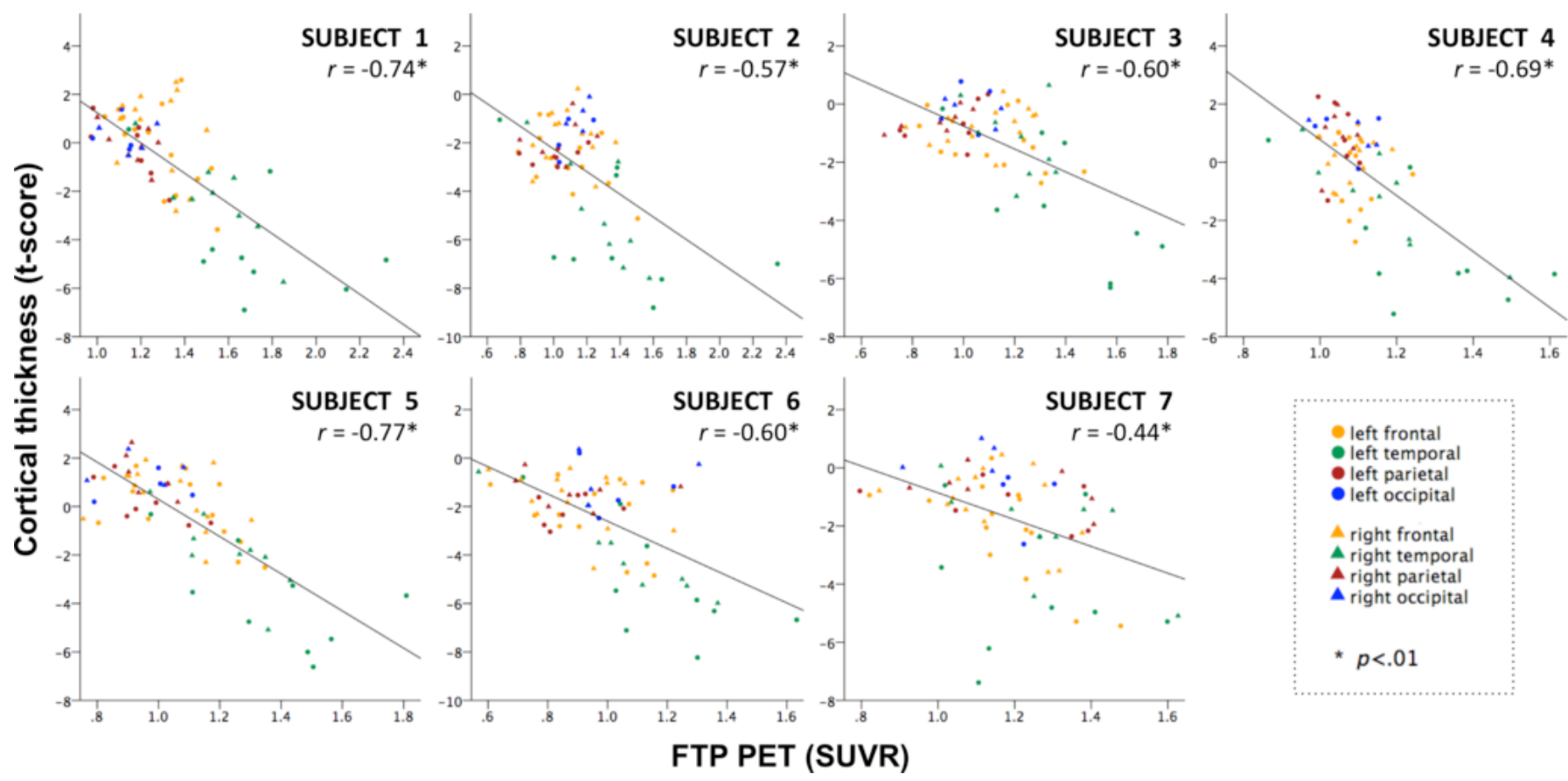

Figure 5 Relationships between cortical atrophy and FTP SUVR measured in 68 cortical regions of interest (ROIs) for each individual patient with sVPPA. In each patient, the magnitude of regional FTP SUVR signal is robustly correlated with magnitude of cortical thickness abnormality (cortical thickness $t$ score for each region compared with a group of cognitively normal participants). Each point on each scatterplot represents the values from a single cortical ROI, colour coded by lobe and separated by hemisphere. Note the consistent presence of the temporal lobe regions in the lower right of each scatterplot. FTP, flortaucipir; SUVR, standardised uptake value ratio; SVPPA, semantic variant of primary progressive aphasia.

molecule(s) to which FTP binds in these svPPA cases is co-localised with neurodegenerative pathology. Recent data from a different tracer, 18F-THK 5351, indicate that one off-target binding site for that tracer is monoamine oxidase $\mathrm{B},{ }^{36}$ which is expressed in astrocytes and has been proposed as a marker for astrocytosis. ${ }^{37}$ To our knowledge, investigation of this issue with FTP has not been reported. Additionally, the relatively low number of subjects limits the extent to which we can reliably identify group differences. Further studies with FTP and other tau PET tracers need to be performed in patients with svPPA who are ultimately followed to autopsy to elucidate binding targets.

Acknowledgements The authors thank the participants and families for their engagement in this research.

Contributors Data collection and analysis: SJM, MQ, KAJ, BCD. Manuscript drafting: all authors.

Funding This study was supported by the National Institute of Deafness and Other Communication Disorders (R01 DC014296), National Institute on Aging (R21 AG051987, R01 AG046396, P01 AG036694,P50 AG00513421), and National Institute of Neurological Disorders and Stroke (R21 NS077059). This research was carried out in whole or in part at the Athinoula A. Martinos Center for Biomedical Imaging at the Massachusetts General Hospital, using resources provided by the Center for Functional Neuroimaging Technologies, P41EB015896, a P41 Biotechnology Resource Grant supported by the National Institute of Biomedical Imaging and Bioengineering (NIBIB), National Institutes of Health. This work also involved the use of instrumentation supported by the NIH Shared Instrumentation Grant Program and/or High-End Instrumentation Grant Program; specifically,grant number(s) S10RR021110, S10RR023043, S10RR023401.

Competing interests None declared.

Ethics approval Partners Human Research Committee.

Provenance and peer review Not commissioned; externally peer reviewed.

Open access This is an open access article distributed in accordance with the terms of the Creative Commons Attribution (CC BY 4.0) license, which permits others to distribute, remix, adapt and build upon this work, for commercial use, provided the original work is properly cited. See: http://creativecommons.org/licenses/by/4.0/

(c) Article author(s) (or their employer(s) unless otherwise stated in the text of the article) 2018. All rights reserved. No commercial use is permitted unless otherwise expressly granted.

\section{REFERENCES}

1 Collins JA, Montal V, Hochberg D, et al. Focal temporal pole atrophy and network degeneration in semantic variant primary progressive aphasia. Brain 2017; 140:457-71.

2 Gorno-Tempini ML, Hillis AE, Weintraub S, et al. Classification of primary progressive aphasia and its variants. Neurology 2011;76:1006-14.

3 Mesulam MM, Dickerson BC, Sherman JC, et al. Case 1-2017. A 70-Year-Old Woman with gradually progressive loss of language. N Engl J Med 2017;376:158-67.

4 Hodges JR, Patterson K, Oxbury S, et al. Semantic dementia. progressive fluent aphasia with temporal lobe atrophy. Brain 1992;115(Pt 6):1783-806.

5 Mackenzie IR, Neumann M, Baborie A, et al. A harmonized classification system for FTLD-TDP pathology. Acta Neuropathol 2011;122:111-3.

6 Mackenzie IR, Neumann M, Bigio EH, et al. Nomenclature for neuropathologic subtypes of frontotemporal lobar degeneration: consensus recommendations. Acta Neuropathol 2009;117:15-18.

7 Alladi S, Xuereb J, Bak T, et al. Focal cortical presentations of Alzheimer's disease. Brain 2007;130:2636-45.

8 Hodges JR, Mitchell J, Dawson K, et al. Semantic dementia: demography, familial factors and survival in a consecutive series of 100 cases. Brain 2010;133:300-6.

9 Chare L, Hodges JR, Leyton CE, et al. New criteria for frontotemporal dementia syndromes: clinical and pathological diagnostic implications. J Neurol Neurosurg Psychiatry 2014:85:865-70.

10 Spinelli EG, Mandelli ML, Miller ZA, et al. Typical and atypical pathology in primary progressive aphasia variants. Ann Neurol 2017:81:430-43.

11 Smith R, Puschmann A, Schöll M, et al. 18F-AV-1451 tau PET imaging correlates strongly with tau neuropathology in MAPT mutation carriers. Brain 2016;139:2372-9.

12 Josephs KA, Whitwell JL, Tacik P, et al. [18F]AV-1451 tau-PET uptake does correlate with quantitatively measured 4R-tau burden in autopsy-confirmed corticobasal degeneration. Acta Neuropathol 2016;132:931-3.

13 Coakeley S, Cho SS, Koshimori Y, et al. Positron emission tomography imaging of tau pathology in progressive supranuclear palsy. J Cereb Blood Flow Metab 2016;271678X:0271678X1668369. 
14 Marquié $\mathrm{M}$, Normandin MD, Vanderburg CR, et al. Validating novel tau positron emission tomography tracer [F-18]-AV-1451 (T807) on postmortem brain tissue. Ann Neurol 2015;78:787-800.

15 Marquié $M$, Normandin MD, Meltzer AC, et al. Pathological correlations of [F-18]AV-1451 imaging in non-alzheimer tauopathies. Ann Neurol 2017;81:117-28.

16 Lowe VJ, Curran G, Fang P, et al. An autoradiographic evaluation of AV-1451 Tau PET in dementia. Acta Neuropathol Commun 2016;4:58.

17 Sander K, Lashley T, Gami P, et al. Characterization of tau positron emission tomography tracer [(18)F]AV-1451 binding to postmortem tissue in Alzheimer's disease, primary tauopathies, and other dementias. Alzheimers Dement 2016;12:1116-24.

18 Ossenkoppele R, Schonhaut DR, Schöll M, et al. Tau PET patterns mirror clinical and neuroanatomical variability in Alzheimer's disease. Brain 2016;139:1551-67.

19 Xia C, Makaretz SJ, Caso C, et al. Association of In Vivo [18F]AV-1451 tau PET Imaging results with cortical atrophy and symptoms in typical and atypical alzheimer disease. JAMA Neurol 2017;74:427.

20 Sapolsky D, Bakkour A, Negreira A, et al. Cortical neuroanatomic correlates of symptom severity in primary progressive aphasia. Neurology 2010;75:358-66.

21 Mesulam MM. Primary progressive aphasia--a language-based dementia. N Engl J Med 2003;349:1535-42.

22 Mormino EC, Betensky RA, Hedden T, et al. Amyloid and APOE $\varepsilon 4$ interact to influence short-term decline in preclinical alzheimer disease. Neurology 2014;82:1760-7.

23 Fischl B, van der Kouwe A, Destrieux C, et al. Automatically parcellating the human cerebral cortex. Cereb Cortex 2004;14:11-22.

24 Shoup TM, Yokell DL, Rice PA, et al. A concise radiosynthesis of the tau radiopharmaceutical, [18F]T807. J Labe/ Compd Radiopharm 2013.

25 Baker SL, Lockhart SN, Price JC, et al. Reference Tissue-Based kinetic evaluation of 18F-AV-1451 for Tau Imaging. J Nucl Med 2017;58:332-8.
26 Greve DN, Salat DH, Bowen SL, et al. Different partial volume correction methods lead to different conclusions: an (18)F-FDG-PET study of aging. Neuroimage 2016;132:334-43.

27 Desikan RS, Ségonne F, Fischl B, et al. An automated labeling system for subdividing the human cerebral cortex on MRI scans into gyral based regions of interest. Neuroimage 2006;31:968-80.

28 Crawford JR, Garthwaite PH, Howell DC. On comparing a single case with a control sample: an alternative perspective. Neuropsychologia 2009;47:2690-5.

29 Johnson KA, Schultz A, Betensky RA, et al. Tau positron emission tomographic imaging in aging and early alzheimer disease. Ann Neurol 2016;79:110-9.

30 Shcherbinin S, Schwarz AJ, Joshi A, et al. Kinetics of the Tau PET Tracer 18F-AV-1451 (T807) in subjects with normal cognitive function, mild cognitive impairment, and Alzheimer Disease. J Nucl Med 2016:57:1535-42.

31 Bigio EH, Wu JY, Deng HX, et al. Inclusions in frontotemporal lobar degeneration with TDP-43 proteinopathy (FTLD-TDP) and amyotrophic lateral sclerosis (ALS), but not FTLD with FUS proteinopathy (FTLD-FUS), have properties of amyloid. Acta Neuropathol 2013;125:463-5.

32 Robinson JL, Geser F, Stieber A, et al. TDP-43 skeins show properties of amyloid in a subset of ALS cases. Acta Neuropathol 2013;125:121-31.

33 Schöll M, Lockhart SN, Schonhaut DR, et al. PET Imaging of Tau Deposition in the Aging Human brain. Neuron 2016;89:971-82.

34 Gomperts SN, Locascio JJ, Makaretz SJ, et al. Tau Positron Emission Tomographic Imaging in the Lewy Body Diseases. JAMA Neurol 2016;73:1334-41.

35 Halabi C, Halabi A, Dean DL, et al. Patterns of striatal degeneration in frontotemporal dementia. Alzheimer Dis Assoc Disord 2013;27:74-83.

$36 \mathrm{Ng} \mathrm{KP}$, Pascoal TA, Mathotaarachchi S, et al. Monoamine oxidase B inhibitor, selegiline, reduces (18)F-THK5351 uptake in the human brain. Alzheimers Res Ther 2017:9:25.

37 Schöll M, Carter SF, Westman E, et al. Early astrocytosis in autosomal dominant Alzheimer's disease measured in vivo by multi-tracer positron emission tomography. Sci Rep 2015;5:16404. 